\title{
Editorial
}

\author{
Anand G. Dabak \\ Wireless Communications Branch, DSPS ReD Center, Texas Instruments, \\ 12500 TI Boulevard, MS 8649, Dallas, TX 75243, USA \\ Email:dabak@ti.com
}

\section{Erik DahIman}

Ericsson Research, 16480 Stockholm, Sweden

Email:erik.dahlman@era.ericsson.se

\section{Giridhar D. Mandyam}

Nokia Research Center, 6000 Connection Drive, Irving, TX 75039, USA

Email: giridhar.mandyam@nokia.com

The past few years have seen worldwide standardization activity of third-generation (3G) wireless systems. Standard bodies such as the Third-Generation Partnership Project (3GPP) and Third-Generation Partnership Project 2 (3GPP2) have been developing 3G standards. The 3G systems are intended to deliver high data rates and are expected to handle data and multimedia applications in addition to voice. Large-scale deployment of $3 \mathrm{G}$ systems is expected to begin during 2002. The first releases of the $3 \mathrm{G}$ standards can deliver data rates up to 384 kbits with widearea coverage and 2 Mbits for indoor environments. Furthermore, evolution of the $3 \mathrm{G}$ systems, delivering peakdata rates of up to 10 Mbits in the downlink direction, are already being standardized. One of the enabling technologies to make these high data rates available is the advance in signal processing. The standards developed by both 3GPP and 3GPP2 already include support for a number of advanced signal processing techniques, such as multiuser detection, smart antennas, and advanced coding techniques.

Concurrently, much recent work in standardization has focused on noncellular technologies such as Bluetooth and wireless local-area network technologies (e.g., HIPERLAN, IEEE 802.11). Such systems are intended to deliver data rates higher than 3G cellular systems under the constraints of limited mobility and indoor operation. These types of technologies and their operating environments present a set of challenges to which advanced signal processing methods are used to address.

The first three papers are on CDMA receiver design for third-generation wireless systems.The paper by K. Hooli,
M. Juntti, M. J. Heikkilä, P. Komulainen, M. Latva-aho, and J. Lilleberg provides an overview of different methods for chip-level equalization in the downlink of wideband CDMA (WCDMA) systems. The paper by S. Chowdhury, M. D. Zoltowski, and J. S. Goldstein concentrates on chip-level equalization for CDMA reception in the downlink using a simplified version of the minimum mean-squared equalizer. The paper by M. J. Borran and B. Aazhang, on the other hand, presents a method for uplink multiuser detection in CDMA systems based on the expectation-maximization (EM) algorithm.

The next two papers cover system level aspects of CDMA. The paper by S.-H. Hwang and D. K. Kim provides an analytical approach to determining the performance of a synchronous CDMA system in the reverse link under the assumption of imperfect power control. The paper by M. Saquib and M. H. Islam describes a method for optimizing CDMA systems that employ multiple transmit and receive antennas in the downlink.

The next two papers describe new methods for improving OFDM system performance. The paper by X. Wang and K. J. R. Liu describes a new method for channel estimation in OFDM systems. The paper by J. D. Terry, J. Heiskala, V. Stolpman, and M. Fozunbal examines the performance benefits from coded bandwidth-efficient modulation approaches in OFDM systems.

The next paper by M. Boyle and A. D. Fagan describes a new approach to maximum likelihood sequence detection (based on the Viterbi algorithm) that addresses the problem of finding the best-desired impulse response (DIR) given the actual channel conditions. Finally, the paper by 
G. D. Mandyam presents a family of block codes that can be used in multiple transmit antenna systems.

Anand G. Dabak Erik Dahlman Giridhar D. Mandyam

Anand G. Dabak received his B.Tech. in electrical engineering from the Indian Institute of Technology, Bombay, India in 1987 and the M.S. and Ph.D. degrees in electrical engineering in 1989 and 1992, respectively, from Rice University, Houston, Texas. After receiving his Ph.D., he worked for Viasat Inc., Carlsbad, California in satellite communications. He joined Texas Instruments

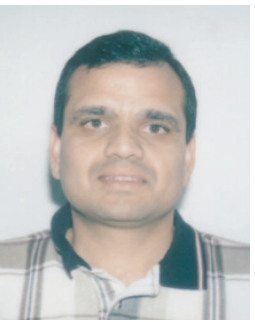
Inc., Dallas, Texas in 1995 and has since then worked on the system and algorithm issues related to wireless communications. Presently, he is a Senior Member of Technical Staff and Manager of mobile wireless research in the DSPS R\&D center of Texas Instruments. He has been involved in the standardization activity in third-generation wireless systems, Bluetooth and IEEE 802.15.3. His work in applying space-time block codes for wideband CDMA systems resulted in the standardization of open loop transmit diversity techniques for third-generation cellular/wireless systems. He holds eight patents in the area of digital communications.

Erik Dahlman received his M.S. in electrical engineering in 1987 and the Ph.D. in Telecommunication in 1992, both from the Royal Institute of Technology in Stockholm. Since 1993, he has been at Ericsson Research, involved in research and development of radio-access technologies for cellular communication. Erik Dahlman was extensively involved in the development and standardization work on wideband CDMA for third-generation mobile communication, both within ETSI and ARIB. Later on, he joined the Third-Generation Partnership Project (3GPP) for the development of the global WCDMA standard. Recently, he has been involved in the standardization of WCDMA release 5, including the support for High Speed Downlink Packet Access. He is also involved in research on radio-access for future-generation cellular systems. Erik Dahlman holds more than twenty patents within the area of wireless communication. In 1998, he was awarded the Inventor of the Year Award of Ericsson, and in 1999, he was awarded the IEEE Vehicular technology Society Jack Neubauer Award for the best system paper.

Giridhar D. Mandyam is the Research Manager of the Wireless Data Access Group at Nokia Research Center, Irving, Texas. He received his B.S. degree Magna Cum Laude in electrical engineering from Southern Methodist University (Dallas, Texas) in 1989 , the M.S. degree in electrical engineering from the University of Southern California (Los Angeles, California) in 1993,

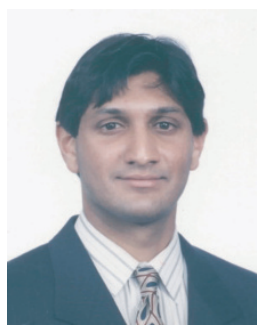
and the Ph.D. degree in the same major from the University of New Mexico (Albuquerque, New Mexico) in 1996. He has worked for several companies on wireless communications equipment, including Qualcomm and Texas Instruments. In 1998, he joined Nokia, where he has worked on standardization and implementation concepts for cdma2000, 1X-EV, and WCDMA. He has authored and coauthored over fourty journal and conference publications and four book chapters. He also holds four US patents in the area of wireless communications technology. 